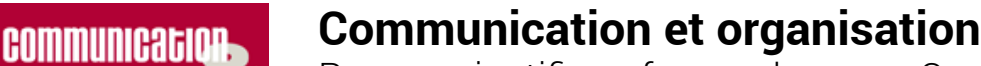

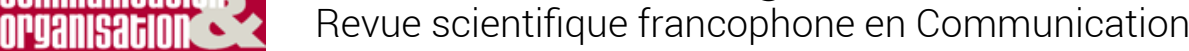

organisationnelle

13 | 1998

Management par projet et logiques

communicationnelles

\title{
La « manipulation » dans la communication
}

Denis Benoît

\section{OpenEdition}

Journals

Édition électronique

URL : https://journals.openedition.org/communicationorganisation/2044

DOI : 10.4000/communicationorganisation.2044

ISSN : 1775-3546

Éditeur

Presses universitaires de Bordeaux

Édition imprimée

Date de publication : 1 mai 1998

ISSN : 1168-5549

Référence électronique

Denis Benoît, « La «manipulation » dans la communication », Communication et organisation [En ligne], 13| 1998, mis en ligne le 26 mars 2012, consulté le 16 février 2023. URL : http://

journals.openedition.org/communicationorganisation/2044; DOI : https://doi.org/10.4000/ communicationorganisation.2044

Ce document a été généré automatiquement le 16 février 2023.

Tous droits réservés 


\title{
La « manipulation » dans la communication
}

\author{
Denis Benoît
}

1 Philippe Breton, dans son ouvrage L'argumentation dans la communication, paru aux éditions La Découverte en octobre 1996, décrit et « fait la promotion » de l'argumentation en tant que communication persuasive «morale»: d'après lui, trois composantes essentielles (ou critères constitutifs) de cet acte de communication « qui vise à faire partager par autrui une opinion» par la «mise en œuvre d'un raisonnement» appartiennent au domaine de l'éthique.

2 Ainsi, pour cet auteur, l'espace de l'argumentation, - notion qui renvoie ici à « un monde dans lequel, dès qu'il s'agit de défendre une opinion la raison l'emporte [...] sur les passions ou l'esthétique ", se démarque - doit se démarquer - de celui de la rhétorique et, plus largement, de celui de la « manipulation », dans le sens où dans ce cadre spécifique, «l'orateur, maître par définition du moment oratoire [...] fait tout pour créer une situation de communication où l'autre est effectivement libre ", (la liberté d'adhésion d'autrui étant directement appréciée en fonction des procédés utilisés par ledit orateur : l'emploi de «figures esthétiques ou émotionnelles » la limite alors que la proposition de Sonnes raisons de croire » la favorise).

3 En fait, d'après P. Breton, à la fois « essentielle au lien social », (elle circonscrit l'« espace public laïque », celui de notre quotidien dont elle constitue la « dynamique essentielle », celle qui donne forme à la matière première de nos croyances, nos opinions, nos valeurs, c'est-à-dire aux «mondes de représentations que nous partageons avec tous les autres humains »), et composante centrale de la citoyenneté en démocratie, (" une société qui ne propose pas à tous ses membres les moyens d'être citoyens, c'est-à-dire d'avoir une véritable compétence à prendre la parole, est-elle vraiment démocratique?»), l'argumentation ne saurait donc « être réduite à une technique » et « fait appel aux piliers de l'éthique »: outre la liberté pour l'auditoire d'adhérer à l'opinion proposée, il est question d'authenticité des arguments utilisés (l'orateur doit partager le point-de-vue qu'il soutient), et de relativité des idées défendues (qui ne sont, au bout du compte, que des opinions). 


\section{«On ne peut pas ne pas manipuler»}

4 C'est, quant à nous, un registre d'action a priori plus vaste que celui que décrit P. Breton, (où il est donc question de "savoir se restreindre au nom d'une éthique »), que nous souhaitons caractériser ici: celui, dès l'abord hétérogène, impur, "malsain», de l'ensemble de la communication "persuasive ", soit celle où la question de l'éthique paraît accessoire, et où le véritable (ou même unique) critère d'évaluation semble être l'adéquation des moyens employés aux fins visées, c'est-à-dire en fin de compte celui de l'efficacité.

5 En fait, sans exactement reprendre à notre compte, dans cette courte étude, une vision du réel où « tout serait pur rapport de pouvoir, exercice de séduction ou usage de techniques manipulatoires ", (et évidemment fortement intéressés comme Philippe Breton par « tout ce qui viendrait soutenir l'idée d'un libre débat entre les hommes »), nous partageons pourtant avec des auteurs aussi différents que Tzvetan Todorov (1983, p.41), Paul Watzlawick (1980 p. 19), François Roustang (1990, p. 44) ou Bernard Cathelat (1988, p. 31), l'idée selon laquelle « il n'y a pas de relation humaine qui ne soit soumise à l'influence [...] il n'y a pas [...] de relation sans manipulation réciproque ».

6 Précisons qu'il n'est en aucun cas question ici, sous le couvert d'une telle affirmation de principe, de faire l'éloge de la "machination », de la «manigance », de la «combine » en tant que telles, ou encore de se livrer à l'apologie de la fraude, de la tromperie ou du mensonge, mais d'affirmer que l'aphorisme "on ne peut pas ne pas manipuler » est directement lié à la formule «on ne peut pas ne pas communiquer», ce qui signifie globalement :

7 - que tout comportement en présence d'autrui - et il n'existe pas de "noncomportement » - produit des effets, pose dans le monde une ou des données par rapport auxquelles l'on devra nécessairement se positionner, et que la communication ne renvoie jamais à une relation « objective » des faits ou avec les faits (ou «neutre» avec autrui) non seulement parce que la réception du message est éminemment subjective, mais aussi parce qu'à la source d'émission le codage de ce même message ne saurait éviter de connoter son objet; le terme "manipulation » est alors entendu dans un sens qui réfère directement à son étymologie latine, manipulus, la gerbe, la botte, la " poignée d'herbes ", ce qui, dans le langage des pharmaciens, a donné le verbe "manipuler », c'est-à-dire "mêler certaines substances chimiques ou pharmaceutiques" qui, par définition, interagissent, ont des effets les unes sur les autres ;

8 - que consciemment, intentionnellement, volontairement, verbalement ou non s'établissent toujours dans les relations humaines des jeux d'influence, des stratégies en rapport avec un ou des « enjeux », un ou des intérêts, (et qu'on ne saurait donc réduire la notion de communication "persuasive » - et limiter le domaine d'investigation désigné par cette expression - à " une volonté humaine [c'est nous qui soulignons] d'établir des relations sociales [...] dont l'objectif premier est de provoquer un changement dans la manière de penser ou le comportement d'autrui ", (Dacheux, 1994, p. 29);

9 - que sachant combien peu est fiable le jugement en matière de lucidité et de sincérité, ( «la lucidité complète sur mes motivations est-elle seulement possible, que je sois manipulé ou manipulateur?», demande par exemple T. Todorov, qui explique que le manipulé reçoit généralement ce qualificatif du fait de son ignorance de l'état dans lequel 
il se trouve et que le manipulateur recueille d'ordinaire cette étiquette car il possède le désir de dissimuler ou de travestir son acte), il est de facto impossible de véritablement distinguer sur le critère a priori décisif de «l'adéquation entre les actes et la conscience qu'on en a » les interactions manipulées de celles qui ne le seraient pas.

Puisque, dans un tel cadre de pensée, il est absurde de se demander comment il serait possible d'éviter toute influence ou manipulation, il convient alors, comme le propose Paul Watzlawick, d'accepter la responsabilité inéluctable de décider pour nous-mêmes de quelle manière «cette loi fondamentale de la communication peut être suivie le plus humainement, le plus honnêtement et le plus efficacement possible ».

11 Ainsi, à proprement parler, c'est bien à partir d'une certaine « philosophie » voire d'une éthique, (sans doute d'une idéologie) que nous conduisons l'exploration de notre propre domaine d'investigations, mais notre conception de base se trouve être fondamentalement différente de celle qu'adopte Philippe Breton dans l'ouvrage susmentionné. Celui-ci, considérant qu'il « existe de nombreux cas [...] où convaincre peut relever avec bonheur, pour l'essentiel, de l'usage de raisonnements qui tiennent compte de l'auditoire dans une situation de libre communication", s'y livre essentiellement à "une réflexion sur les conditions d'une parole argumentative à l'opposé de la rhétorique et de la manipulation ». Quant à nous, nous postulons qu'un certain nombre de règles (principalement celle selon laquelle l'on ne peut pas ne pas " manipuler ») viennent toujours - argumentation ou non - contraindre ou limiter la liberté des situations de communication (et donc des communicants), et que si l'ouvrage de Breton est indéniablement utile (en particulier en inscrivant explicitement l'argumentation dans le champ des sciences de l'information et de la communication ou en permettant de "saisir l'acte d'argumenter dans toute sa dynamique de communication»), l'essai qui cherche notamment à distinguer argumentation et rhétorique peut peut-être rester vain pour l'essentiel.

Liés à cette différence conceptuelle de base, (ou lui servant de support), ce sont deux points spécifiques de l'exposé de $P$. Breton qui nous paraissent pouvoir être discutés.

\section{Argumentation versus rhétorique}

D'une part, en effet, l'argumentation que développe cet auteur sur l'objet « argumentation » en vient à considérer - bien légitimement - l'existence d'un « doute [...] sur l'existence d'un acte de communication autonome qui permettrait de transférer, uniquement par le raisonnement, une opinion de l'un vers l'autre ", (les situations de communications étant composées de "couches insécables [...] toute la richesse de l'activité humaine y est toujours présente simultanément»). Cette argumentation est alors amenée à employer l'idée de " dominante » : " tout en impliquant la palette entière des réactions humaines, l'acte d'argumentation suppose une dominante de raisonnement et, parallèlement, une minoration de l'appel aux sentiments, au pouvoir ou même à la démonstration ».

Or, nous ne sommes que peu enthousiaste à l'idée de devoir classer dans le genre ainsi caractérisé certaines formes désignées comme argument, telles le recadrage ou la métaphore. Considérer par exemple qu'en situation de communication l'utilisation d'une métaphore « mise au service de la défense d'une thèse ou d'une opinion » réfère plus à la «mise en œuvre d'un raisonnement" qu'à un «appel aux sentiments » nous paraît 
controversable. C'est ainsi qu'Olivier Reboul (1984, pp. 32-33 ; 1991 p.5, 98, 119, 188), s'il distingue bien des moyens de persuader "plus rationnels » et d'autres "plus affectifs", renonce pourtant définitivement à « séparer nettement les genres » (selon l'expression de Breton pour lequel «le bon usage de l'argumentation implique [...] une rupture avec la rhétorique classique »), c'est-à-dire à disjoindre d'une certaine façon le "persuader » du «convaincre ». Il évite ainsi, peu ou prou, de dissocier - dissociation que la notion de « dominante » a, par la bande, tendance à réintroduire -, l'homme « être de croyance et de sentiment » de l'homme «être d'intelligence et de raison »: d'après Reboul, l'essence du discours persuasif c'est justement «l'articulation des arguments et du style dans une même fonction ", c'est-à-dire "l'intersection de la littérature et de la persuasion ", là où "les trois fonctions de plaire, d'instruire et d'émouvoir sont présentes ensemble et chacune par les autres». En tant que «figure» (et non que simple «condensé d'argument» comme la réduisent également Olbrechts-Tyteca et Perelman selon Reboul), c'est-à-dire en tant que procédé « qui montre le mieux le joint entre le style et l'argumentation », (soit l'idée que "l'esthétique et le persuasif sont indissolublement liés »), la métaphore apparaît alors toujours chez ce dernier auteur comme susceptible à la fois de "nous éclairer, nous charmer, nous émouvoir ». Et de même pour le «recadrage » que Paul Watzlawick, Richard Fisch et John Weakland (1975, p. 116) définissent explicitement comme modifiant "le contexte conceptuel et/ou [c'est nous qui soulignons] émotionnel d'une situation »: les moyens de fixer un nouveau cadre, de construire un autre "réel de référence", apparaissent renvoyer très souvent massivement au pathos et à l'ethos des protagonistes, comme dans l'exemple cité par P. Breton (1996, p. 60) où le recadrage obtenu par Tom Sawyer, le jeune héros de Mark Twain, procède au moins autant de l'« impression » qu'il réussit à transmettre à ses camarades que du raisonnement qu'il leur propose : le « pas de côté » qu'il réalise au moment stratégique pour recadrer son «travail» en «loisir désirable" s'apparente à une véritable "figure de style», (rappelons qu'étymologiquement la notion de «figure » renvoie d'abord au «mouvement type de l'athlète ou du danseur »), et est au moins aussi efficace que le raisonnement qu'il utilise, si bien qu'on ne sait, au bout du compte, exactement dire lequel du raisonnement ou de la figure est prépondérant ou dominant dans le résultat obtenu.

\section{Contenu versus relation}

15 Fidèle, donc, à l'idée selon laquelle « il faut refuser le choix mortel entre une rhétorique de l'argumentation et une rhétorique du style », «l'une ne va jamais sans l'autre » (selon Reboul, 1991, p. 98), nous insistons d'autre part sur l'idée que toute argumentation - soit la transmission d'une opinion mise en forme, autrement dit celle d'un "contenu » - ne peut s'effectuer que dans le cadre d'une « relation » liant l'orateur à l'auditoire.

L'on sait en effet qu'un axiome célèbre proposé, à la base de leur réflexion, par certains auteurs de la «nouvelle communication «(Winkin, 1981), énonce que la «relation englobe le contenu » (Watzlawick, Helmick Beavin, Don D. Jackson, 1972, p. 52) ; c'est-àdire que, pour eux, dans toute communication ces deux aspects sont obligatoirement liés, le premier "renseignant» le second à la fois en précisant «la manière dont on doit entendre le message » et en spécifiant une certaine vision de soi-même et d'autrui. Sont de la sorte "mises en jeu » à l'occasion de la transmission du message lui-même des assertions du type «Voici comment je me vois », « Voici comment je vous vois », «Voici 
comment je vous vois me voir ", etc., (propositions évidemment liées au pathos et à l'ethos propres à chacun des communicants) ${ }^{1}$.

17 Ainsi, si la "métacommunication " (la communication sur la communication) constitue bien «une sorte de rationalisation de la relation, de traduction de celle-ci dans le domaine argumentatif» (selon l'expression de P. Breton), elle est aussi, dans une autre acception, la «relation » elle-même et représente la base ou le contenant inéluctable de tout échange de message, et par là même de toute argumentation.

Et c'est exactement à ce point que le bât blesse car si l'on ne peut l'éviter, c'est le plus souvent inconsciemment (ou encore «analogiquement», non-verbalement) que la métacommunication est spécifiée, déterminée par les communicants, (Watzlawick, Helmick Beavin, Don D. Jackson, 1972, p. 50). De ce fait, quand bien même l'orateur souhaite ne pas miser - ou, en tout cas, « minorer », « jouer » aussi peu que possible - sur la relation qu'il entretient avec son auditoire pour « faire passer » l'argument (séduction), et qu'il cherche du mieux possible à laisser «se dérouler le chemin plus long et plus difficile opinion/orateur/argument/auditoire ", (c'est-à-dire la caractéristique même de l'argumentation selon Breton), c'est pourtant le plus souvent à un niveau inconscient, très rarement verbal, c'est-à-dire non explicité par les communicants, que finit tout de même par se régler ou se réguler l'échange. L'effet, "pervers " pourrait-on dire, d'une telle régulation est que moins la relation est prise en compte (notamment lorsque l'on cherche à la minorer, à la faire passer au second plan, pour se concentrer sur le contenu) et plus celle-ci est agissante ou prégnante parce qu'inaperçue (ou peu ou mal perçue), et donc non-maîtrisée (ou peu ou mal maîtrisée). Ainsi, des communicants qui ne tiendrait aucun compte des deux différents niveaux de la communication, ou qui les jaugeraient non-pertinemment en confondant le contenu et la relation - en s'efforçant par exemple de dissiper un désaccord au niveau du contenu ou en argumentant sur ce plan alors que l'enjeu se situerait au niveau relationnel (par exemple, des interactants qui discuteraient inlassablement, en " coupant les cheveux en quatre ", de la valeur de vérité d'un certain énoncé alors que la véritable question concernerait le droit même de l'un par rapport à l'autre d'émettre cet énoncé) -, de tels communicants ne pourraient qu'aboutir à un culde-sac dans leur échange; impasse qui pourrait se manifester par une «redondance monotone de pseudo-désaccords », (selon Watzlawick, Helmick Beavin, Don D. Jackson, 1972, pp. 79-82).

Dès lors, on voit que la « forme » ne peut pas ne pas influencer le «fond »; que les règles liées aux propriétés du processus de la communication (de la «transmission») limitent toujours la liberté des communicants; et plus qu'avoir un impact, un effet sur le contenu du message lui-même, la forme de la transmission, l'« esthétique ", apparaît comme partie intégrante du fond : quand bien même il utiliserait un raisonnement impeccable (contenu), celui qui, par exemple, argumente en "symétrisant » inconsciemment excessivement l'échange peut de facto le transformer en confrontation, en polémique (relation) du type «Mais non... Mais si...» propre à contrarier sinon à faire capoter tout projet de persuasion, de conviction ou de « changement ».

Ainsi, si « argumenter n'est pas convaincre à tout prix »-ce qui, pour P. Breton, suppose « une rupture avec la rhétorique au sens où celle-ci n'est pas regardante sur les moyens de persuader » - nous dirons quant à nous que convaincre en argumentant reste tout de même tributaire d'un " prix à payer » fort conséquent: le raisonnement ou les «bonnes raisons » destinés à «faire croire » sont eux-mêmes toujours modelés par le processus de 
leur transmission, c'est-à-dire extrêmement dépendants de l'établissement et du maintien d'une certaine « relation » - qui vient « d'abord » (Bougnoux, 1995, p. 35).

\section{La raison avec l'émotion} leur Traité de l'Argumentation est qu'« entre la démonstration scientifique et l'arbitraire des croyances, il existe une logique du vraisemblable, qu'ils nomment l'argumentation ». De la sorte, pour convaincre et persuader (ou se convaincre et se persuader), il existe une voie moyenne entre la rigueur «scientifique » et le trouble, la confusion des passions, (l'entendement et la volonté, l'esprit de géométrie et l'esprit de finesse, la raison et le cœur, selon Pascal).

rtant, pour le même Reboul, la démarche que proposent ces auteurs est fondamentalement incomplète: «si le TA décrit à merveille les stratégies de l'argumentation, il méconnaît les aspects affectifs de la Rhétorique, le delectare et le movere, le charme et l'émotion, pourtant essentiels à la persuasion ".

r, notre propre approche de la communication persuasive va exactement de pair avec cette dernière affirmation, et si l'on recherchait une base fondamentale sur laquelle s'échafaude notre démarche l'on pourrait la trouver dans l'idée (ou l'option) de placer raison et passion, "logique " et sentiment, argumentation et séduction sur un plan équivalent: quelles que soient les situations de relations humaines les deux aspects revêtent une importance similaire, et plutôt que, peu ou prou, chercher à les distinguer, il convient au contraire d'apprécier leurs rôles complémentaires et leurs interactions mutuelles, (ce qui, en un sens, rejoint le propos de Philippe Breton qui, notamment, suggère de réfléchir à la façon dont «l'appel aux sentiments» peut, dans l'argumentation, être "mis au service» du raisonnement "sans pour autant le paralyser ». arriverait à une conception spécifique de la notion de « raison ».

En effet, il semble que ce dernier terme, qui autrefois renvoyait généralement à un véritable principe d'explication absolu et immuable, à une " fonction de pensée correcte » s'opposant à la connaissance imparfaite et illusoire, apte à nous faire «dépasser les apparences et atteindre les réalités ", ne puisse, au point actuel de la réflexion épistémologique, « se définir utilement que dans un contexte » - et Raymond Boudon a pu parler à son sujet de terme "polythétique », (1990, p. 317-369) : il semble que la raison doive être appréhendée comme une «figure d'équilibre provisoire de l'imagination créatrice ", qui, « loin d'être une forme définitivement fixée de la pensée », demeure une « incessante conquête », (Granger, 1984, pp. 7,10,126).

Dans le prolongement direct d'une telle analyse, nous considérons, à l'instar de Paul Watzlawick qui se fonde sur la théorie de l'asymétrie hémisphérique cérébrale (1980, pp. 19-20,45-46,52-53), que l'idée-force qui veut que la raison soit «la plus haute faculté de l'homme » - celle grâce à laquelle celui-ci peut « saisir la vraie réalité, celle qui existe objectivement « dehors » - doit être considérée comme renvoyant à un mythe. Car « l'on peut seulement parler d'interprétations de la réalité, d'images du monde et non de réalité en tant que telle»: c'est là l'idée-clef du «constructivisme radical ", (Watzlawick, 1988, passim). Or, c'est à l'hémisphère droit du cerveau, spécialisé dans la perception des 
« totalités ", des structures complexes (Zaidel, 1984 ; Sergent, 1990), et compétent pour la construction des classes logiques et donc pour la formation des concepts, que revient sans doute la fonction de "synthétiser [...] en une image notre expérience du monde », (alors qu'il est probablement dévolu à l'hémisphère gauche «de rationaliser cette image, de diviser cette totalité [...] en sujet et objet »).

Ces « images du monde", construites "par et à travers la communication", sont donc principalement conçues dans un langage "archaïque et peu développé " (propre à l'hémisphère droit), langage où dominent « l'image, l'analogie, et par conséquent aussi, la mémoire des événements et des sentiments et sensations qui les accompagnaient", (Watzlawick, 1980, pp. 33-34), en fait, un langage qui renvoie à l'ensemble des ressources de la rhétorique (qui est d'ailleurs considérée par Paul Watzlawick comme «le véritable précurseur des recherches actuelles sur la communication »).

Dans ces conditions, l'on ne peut manquer d'estimer que, raisonnement et émotions étant étroitement reliés, toute conviction (ou toute démarche visant à convaincre, c'est-à-dire à modifier ou à structurer chez soi-même ou chez autrui une certaine image du monde) comprend quasi obligatoirement (au moins dans un premier temps, celui de la construction de l'image) une composante cruciale liée à l'esthétique, à l'émotion, à la «sensation ", à l'« affect », bref à la forme et à la « relation " ; et les travaux actuels du neuropsychologue Antonio Damasio (1994, passim), qui tendent à montrer «que les mécanismes neurologiques des émotions sont liés à ceux de la décision", semblent d'ailleurs fortement accréditer la thèse selon laquelle « sans émotions nos raisonnements les mieux menés n'aboutiraient à aucun choix. Or, un homme sans préférences ne saurait être rationnel », (Journet, 1997, p. 17) : pour lui, dans son ouvrage L'erreur de Descartes - La raison des émotions, "l'absence d'émotions et de sentiments empêche d'être vraiment rationnel »...

Dès lors, comme nous l'écrivions dans un texte destiné à inventorier les contenus et référents possibles du concept de manipulation en matière de relations humaines (Benoit, 1990, p.18), tout un aspect de l'homme relève de l'«irrationnel", de l'affectif, de l'« imaginaire » qui, au moins au même titre que la logique, l'analyse, la raison, constituent un moyen d'appréhension (ou de construction) de ce que l'on nomme « la réalité ». Et si, au nom d'une éthique déterminée, l'on peut indubitablement combattre le conditionnement ou la colonisation de cet imaginaire, réalisé par exemple à grande échelle par le réductionnisme de certaines publicités et le "matraquage » permanent du public, (Brune, 1985, passim), il semble en revanche qu'il ne soit définitivement pas pertinent de considérer la rhétorique et ses techniques comme perversion, corruption ou affaiblissement de la raison: pour O. Reboul (1984, p. 120), la rhétorique est bien un " mélange inextricable [c'est nous qui soulignons] d'affectif et de rationnel. Certes, mais ce mélange, c'est l'homme lui-même. Il est vain de croire que nous puissions jamais penser et décider de façon purement rationnelle ». Ni pertinent de se demander comment éviter toute "manipulation ", (dans l'acception du terme que nous avons retenue, c'est-à-dire où celui-ci réfère à tout autre chose qu'à la tromperie, la fraude, la désinformation, toujours illégitimes); manipulation à laquelle nous semblons, comme par nature, irrémédiablement voués. 


\section{L'homme « rationnel » versus l'homme « rationalisant »}

30

Ainsi, en fonction de l'ambiguïté même du concept de « raison » - paradoxalement, donc, la raison implique ou contient l'émotion (ou vice-versa) - plutôt que, peu ou prou, opposer «rhétorique des sentiments» et «argumentation à dominante de raisonnement ", c'est-à-dire distinguer la persuasion irrationnelle ("séduction », «manipulation») d'une autre essentiellement fondée sur la transmission de «bonnes raisons «de croire (susceptibles d'entrainer la «conviction»), il nous parait plus judicieux, au moins concernant notre étude dédiée à l'ensemble de la communication "persuasive ", de différencier, à l'instar des psychosociologues Jean-Léon Beauvois et Robert-Vincent Joule (1988), «l'homme rationnel» de «l'homme rationalisant» en conférant à ces deux expressions un sens bien spécifique :

- l'homme « rationnel » c'est celui qui, même s'il est évidemment sensible à l'émotion et au plaisir, "pèse ses raisons ", «infère ", agit "en fonction ", tire les conséquences d'un raisonnement logique, en bref, un homme qui « pense avant d'agir »;

- alors que l'homme "rationalisant», c'est, pour employer une formule lapidaire, celui « qui agit puis qui pense » : ce n'est pas parce qu'il soutient telle position qu'il agit de telle manière, mais parce qu'il a agi (comme il a été amené à le faire) qu'il va adopter telle position.

La prise en compte d'une telle distinction amène alors à redéfinir la notion de persuasion, et conférer à ce terme une signification beaucoup plus large que celle du sens commun : dans un tel cadre de pensée, il réfère à l'ensemble des techniques qui «mènent au consentement", l'ensemble des «facteurs qui poussent un individu à accepter une proposition », (Cialdini, 1990, p. 7).

34 Dès lors, étudier la communication « persuasive » c'est (notamment) prendre à la fois en considération deux types différents de stratégies.

Le premier type renvoie au « système rhétorique » et à sa partie fondamentale, la théorie de l'argumentation, (Plantin, 1996, passim): le sujet-émetteur, utilisant l'instrument naturel qu'est le langage, cherche à persuader - à la fois par l'« expliquer », le " plaire » et l'« émouvoir "-, c'est-à-dire à obtenir l'adhésion du sujet-récepteur ; soit, en pratique, à modifier les attitudes de celui-ci à l'égard d'un objet déterminé. L'émetteur escompte évidemment le plus souvent que cette transformation des attitudes du récepteur va engager un renouvellement de ses conduites à l'égard de cet objet, (comme dans un procès d'assise, une plaidoirie, par un certain nombre d'arguments, va agir sur l'intime conviction des jurés; conviction qui sera - l'on n'en doute pas - à l'origine du comportement de vote des dits jurés).

36 Avec ce premier type de stratégies, il s'agit donc de "travailler» au "niveau idéologique » - c'est-à-dire au niveau des « cognitions » : savoirs, croyances, valeurs ou opinions - par l'action directe du langage, l'hypothèse implicite étant qu'une "modification des attitudes aura pour conséquence l'apparition de nouvelles conduites ", (Beauvois et Joule, 1981, p. 21).

Dans le second type de stratégies, la "persuasion »-comprise donc au sens le plus large du terme - s'obtient par un ensemble de « techniques comportementales ». Il n'est là plus question d'utiliser le seul langage verbal pour argumenter et séduire aux fins de modifier une attitude, mais d'obtenir certains actes ou certaines décisions - dits comportements « extorqués » - qui vont «engager » celui qui les émet, rendant ainsi plus probable la 
réalisation du ou des comportements souhaités - dits comportements « cibles ». Il s'agit en fait ici d'obtenir une "soumission librement consentie»: l'expression traduit le paradoxe de sujets « persuadés » ou, plus exactement, manipulés par certaines techniques " comportementales »; ces sujets se "soumettent », c'est-à-dire qu'ils réalisent pour autrui (le manipulateur) certains comportements qu'ils n'auraient pas réalisé de leur propre chef; mais cette soumission est effectuée dans un contexte de liberté affirmée, rien ne leur est imposé et ils agissent « en toute liberté ».

Considérée par certains comme un «nouveau paradigme» en psychologie sociale, la "soumission librement consentie» peut être définie sur le plan théorique, comme « l'étude des conséquences des actes sur le plan du comportement et des idées » et sur le plan pratique, comme «l'étude des techniques comportementales qui permettent de peser sur les comportements d'autrui et de l'influencer dans ses idées ». Par rapport à une stratégie de persuasion comprise au sens strict (rhétorique), elle "présuppose un renversement du rapport entre l'attitude et le comportement " : l'attitude apparait alors non plus comme un préalable à la conduite - je pense puis j'agis en fonction de mon attitude - mais sous la forme d'une "sécrétion de la conduite»: c'est l'attitude qui "s'ajuste " à la conduite et non l'inverse. Dans cette optique, l'attitude, ou plutôt le changement d'attitude est donc la conséquence non d'un raisonnement préalable ( « choix rationnel ») mais de la réalisation d'une conduite et de sa « rationalisation », (c'est-à-dire l'activité qui consiste à justifier a posteriori une conduite, un comportement par une nouvelle attitude, une nouvelle valeur) ${ }^{3}$.

Beauvois et Joule illustrent cette seconde stratégie d'influence par le "schéma du démarcheur à domicile » : celui-ci, avant toute conviction de son client potentiel, va chercher à en obtenir certains comportements. Par exemple, si pour une raison ou une autre le démarcheur réussit à pénétrer dans l'appartement dudit prospect, l'attitude de ce dernier pourra être largement modifiée (dans un sens positif) à l'égard du produit proposé, et ceci non pas à cause d'une quelconque communication persuasive (discours argumenté) concernant ce produit mais, par exemple, grâce à la rationalisation qui veut que puisque le client potentiel a admis quelqu'un à pénétrer chez lui ce quelqu'un doit être intéressant, et, par définition, faire des propositions intéressantes.

\section{Un panorama de la recherche en communication persuasive}

Une telle dichotomie - l'homme « rationnel »/l'homme « rationnalisant »- nous permet en fait de circonscrire un champ de recherche que nous centrons autour de l'idée de «manipulation »: pour nous, nous l'avons déjà précisé, si l'on ne peut pas ne pas communiquer, l'on ne peut pas ne pas manipuler, ce qui signifie que toute communication produit des effets - elle «manipule " au sens étymologique et instrumental du terme - et, consciemment ou non, s'établissent toujours dans les relations humaines des « jeux » d'influence.

Etudier ces jeux - au sens de systèmes de relations gouvernés par des règles - revient :

- premièrement, à partir d'une approche "anthropologique ", (Winkin, 1996, passim): exploration, en amont, des « règles » de la communication en fonction des cultures et des contextes; analyse des processus psychosociaux «par lesquels la société et les individus s'engendrent corrélativement et sans lesquels [...] il n'y aurait ni individu ni société ", 
(Huisman, 1983, p. 58) ; en fait, analyse du processus communicationnel dans lequel sont engagés les interactants, soit un "processus permanent à plusieurs niveaux ", hypercomplexe car renvoyant à des aspects physiques, biologiques, psychologiques, sociologiques, ethnologiques, etc., imbriqués en systèmes autoréflexifs, à envisager en termes « de contextes multiples et de systèmes circulaires "; argumentations par lesquelles on nous convie à adhérer à une opinion plutôt qu'à une autre », (il s'agit ici, comme l'explique Perelman, de l'examen des « structures argumentarives ", c'est-à-dire des « moyens discursifs » mis en œuvre pour obtenir un certain état de conscience que l'on nomme "adhésion »: par exemple, l'étude des arguments quasi logiques, qui tirent leur force persuasive de leur ressemblance avec la démonstration) ; mais type qui renvoie aussi à l'observation expérimentale de l'efficacité comparée des diverses formes de communication, (il s'agit là d'études de psychosociologie ou de psychologie expérimentale : par exemple, à la question « faut-il, pour être efficace, qu'une communication présente une argumentation contraire au point de vue qu'elle défend afin d'en minimiser la portée ", les résultats des travaux d'Hovland, Lumsdaine et Sheffield (1949) permettent de répondre que les communication « pour/contre » sont plus efficaces lorsque l'on a notamment à faire avec des auditeurs de niveau culturel élevé) ;

- un second type qui renvoie au paradigme de la « soumission librement consentie» : dans ce cadre, les stratégies de "persuasion» étudiées seront celles qui reposent "sur l'obtention de décisions [ou de comportements] susceptibles d'affecter dans un sens souhaité, les choix, les opinions ou les comportements à venir ».

En fait, l'on peut dire qu'il s'agit, dans notre recherche, de déceler ce que l'on pourrait nommer les "structures persuasives" consubstantielles aux différentes formes de communication, c'est-à-dire les ingrédients qui, qu'il s'agisse de communication sociale ou de communication interpersonnelle, permettent d'emporter la conviction, le consentement, l'adhésion. De faire penser, croire ou agir.

47 C'est alors sur tout un corpus de situations différentes que porte la recherche: par exemple, que fait un vendeur de grande surface ou de boutique de proximité lorsqu'il réalise une vente, quelles sont les techniques très précises qu'il met en œuvre consciemment ou non? Quels sont les comportements adoptés par une présentatrice de téléachat - intentionnellement ou non - pour fidéliser une clientèle à ce type de commerce? Que fait et que dit exactement un homme politique lorsque dans une émission de télévision réalisée en direct, il réussit à convaincre, chiffres à l'appui, une partie de l'opinion? Quelles sont les mises en scène de l'information qui permettent le mieux l'influence et la persuasion du public? Quels sont les modes de fonctionnement et selon quels systèmes le pouvoir se répartit-il dans des groupes et des organisations qui vont de la famille à la communauté internationale, etc?

Il nous appartient, dans ces situations, d'étudier selon le canevas théorique proposé l'homme "rationnel »/l'homme "rationalisant », soit l'opposition entre l'homme qui, même s'il n'obéit pas à une rationalité cartésienne se réfère à une logique du vraisemblable (il pense avant d'agir) versus celui qui rationnalise ce que les circonstances lui ont imposé comme comportement - d'étudier (en fonction de prémisses «anthropologiques ») les arguments utilisés, les figures de style employées, en fait les différentes techniques de "manipulation», le plus souvent étroitement imbriquées, 
consciemment ou inconsciemment mises en œuvre: jeux sur la relation, recadrages, sophismes, emplois du langage du récepteur, confusions-saupoudrages, pieds-dans-laporte, amorçages et autres escalades d'engagement, etc.

Pour conclure ce rapide panorama, nous dirons que nous concevons la recherche en communication persuasive comme une "recherche-action", dans la mesure où, à terme, il est question de mettre en pratique les éléments théoriques élaborés ou réfléchis dans l'abstrait, c'est-à-dire, avant tout, de faire preuve (autant que possible) de lucidité, et ce quelque soit la situation: c'est en connaissant les pratiques et les techniques de communication persuasive que l'on peut à la fois se prémunir efficacement contre la persuasion et la manipulation et, le cas échéant, persuader ou manipuler soi-même.

\section{BIBLIOGRAPHIE}

BEAUVOIS Jean-Léon, Joule ROBERT-Vincent, « La psychologie de la soumission », in La Recherche, $\mathrm{n}$ - 202, 1988, pp. 1050-1057.

BEAUVOIS Jean-Léon, JOULE Robert-Vincent, Soumission et idéologies, Paris, P.U.F., 1981.

BENOIT Denis, «Contenus et référents du concept de manipulation en matière de relations humaines ", in Medianalyses 7, Presses du Centre du XX Siècle, Université de Nice / Sophia Antipolis, 1990, pp. 7-19.

Bougnoux Daniel, La communication contre l'information, Paris, Hachette, 1995.

BOUDON Raymond, L'art de se persuader - des idées douteuses, fragiles ou fausses, Paris, Fayard, 1990.

BRETON Philippe, L'argumentation dans la communication, Paris, La Découverte, 1996.

BRUNE François, Le bonheur conforme, Paris, Gallimard, 1985.

CATHELAT Bernard, EBGUY Robert, Styles de pub - 60 manières de communiquer, Paris, Les Editions d'Organisation, 1988.

CIALDINI Robert, Influence et manipulation, Paris, First, 1990.

DACHEUX Eric, Les stratégies de communication persuasive dans l'union européenne, Paris, L'Harmattan, 1994.

DAMASIO Antonio, L'erreur de Descartes - La raison des émotions, Paris, Editions Odile Jacob, 1995.

GRANGER Gilles-Gaston, La raison, Paris, PUF, 1984.

HUISMAN Denis, Le dire et le faire - Essai sur la communication efficace, Paris, CDU et SEDES, 1983.

JOURNET Nicolas, « Les émotions de A à Z », in Sciences humaines, nº 68,1997, pp. 16-18.

PERELMAN Chaïm, OlBreChTZ-Tyteca Lucie, Traité de l'argumentation, Bruxelles, Editions de

l'Université de Bruxelles, 1992.

PLANTIN Christian, L'argumentation, Paris, Seuil, 1996.

REBOUL Olivier, La rhétorique, Paris, PUF, 1984. 
REBOUL Olivier, Introduction à la rhétorique, Paris, Seuil, 1991.

ROUSTANG François, Influence, Paris, Editions de Minuit, 1990.

SERGENT Justine, « Les dilemmes de la gauche et de la droite - Opposition, cohabitation ou coopération?», in SERON Xavier, Psychologie et cerveau, Paris, PUF, 1990.

THUILUER Pierre, « Du rêve à la science : le serpent de Kekulé », in La Recherche, $\mathrm{n}^{\circ}$ 175,1986, pp. 386-390.

TODOROV Tzvetan, « Eloquence, morale et vérité », in Les manipulations, Le genre humain 6., Arthème Fayard et Editions Complexe, 1983, pp. 26-41.

WATZLAWICK Paul, Le langage du changement - Paradoxes et psychothérapie, Paris, Seuil, 1980.

watzlawick Paul, (sous la direction de), L'invention de la réalité - Contributions au constructivisme, Paris, Seuil, 1988.

Watzlawick Paul, Helmick-Beavin Janet, Don D. Jackson, Une logique de la communication, Paris, Seuil, 1972.

WATZLAWick Paul, Fisch Richard, WeAKLAND John, Changement - Eléments de communication thérapeutique, Paris, Seuil, 1975.

WINKIN Yves, La nouvelle communication, Paris, Seuil, 1981.

WINKIN Yves, Anthropologie de la communication, Bruxelles, De Bœck, 1996.

ZAIDEL Dahlia, « Les fonctions de l'hémisphère droit », in La Recherche, n 153,1984, pp. 332-340.

\section{NOTES}

1. Et Paul Watzlawick - «sans preuve objective »-considère que dans une séquence de communiciation seul le cinquième concerne le contenu, tout le reste étant dévolu a la définition de la relation : il s'agit la de « reconstruire sans cesse le concept de soi, a offrir ce concept de soi aux autres pour ratification, et à accepter ou rejeter les offres que font les autres de leur concept d'eux-mêmes» .

2. Le domaine de la science pure est lui-même concerné : voir l'article de Pierre Thuillier (1986) où l'auteur, exposant une controverse concernant l'existence et le rôles «des éléments « irrationnels » dans la recherche scientifique », cite le chimiste allemand August Kekulé (dont la fameuse contribution sur la structure du benzène -fondamentale pour le développement de la chimie organique- a, selon les propres dires de ce scientifique, été initiée à partir d'un simple rêve au coin $\mathrm{du} \mathrm{feu}$ ) : «Apprenons a rêver. [...] Mais gardons-nous de rendre publics nos rêves avant qu'ils n'aient été mis a l'épreuve par notre esprit bien éveillé ".

3. Précisons qu'il ne s'agit pas d'une reconsidération effectuée à la lumière de nouvelles informations, mais d'une justification sans informations supplémentaires. 


\section{RÉSUMÉS}

Toute communication produit des effets : elle manipule « au sens instrumental du terme. Dès lors, aussitôt que l'on communique l'on manipule, et c'est bien cet axiome fondamental qui doit être posé à la base de toute étude exhaustive en la matière, et notamment des recherches en communication "persuasive", soit celles qui traitent à la fois de l'«efficacité" et de l'« efficience » de la communication, c'est-à-dire à la fois des effets voulus sur autrui par l'auteur d'un message et de ceux que celui-ci n'a pas « consciemment » recherchés. Ainsi l'essai qui tente de distinguer argumentation et rhétorique n'apparaît pas parfaitement satisfaisant dans la mesure où toute communication d'arguments s'établit sur le substrat d'une « relation » implicite qui conditionne l'échange, et que « fond » et « forme » sont indissolublement liés.

Every communication produces effects: it «manipulates » in the instrumental acceptance of the word. Thus, as soon as we communicate we manipulate, and this is indeed this basic " axiome " that must be put at the very base of any extensive study in this field, and particularly as far as "persuasive communication» is concerned, that is to say studies regarding at the same time " efficiency " and "effectiveness " in communication, that is to say both the effects meant on somebody by the person uttering the message and those he didn't knowingly mean, therefore, attempting to distinguish between argumentation and rhetoric doesn't seem to be rotally satisfying in the extent that every communication of arguments establishes on the basis of an implicit « relationship » which the exchange depends on, and « content » and « form » are very closely linked.

\section{AUTEUR}

\section{DENIS BENOÎT}

Docteur en Sciences de l'Information et de la Communication, Maître de conférences à l'Université de Poitiers, responsable coordonnateur d'une Introduction aux Sciences de l'Information et de la Communication (ouvrage collectif publié aux Editions d'Organisation en 1995). Le thème essentiel de la recherche concerne la communication « persuasive », c'est-à-dire, en amont, le lien social, et aval, les tactiques, stratégies, « techniques » de communication permettant aux acteurs d'atteindre certains objectifs en matière de relations humaines. Un ouvrage, La manipulation dans la communication, est en préparation aux éditions Nathan. 